\title{
Optimized joint timing synchronization and channel estimation for communications systems with multiple transmit antennas
}

\author{
Te-Lung Kung* and Keshab K Parhi
}

\begin{abstract}
This paper proposes a joint timing synchronization and channel estimation scheme for communications systems with multiple transmit antennas based on a well-designed training sequence arrangement. In addition, a generalized maximum-likelihood (ML) channel estimation scheme is presented, and this one-shot scheme is applied to obtain all channel impulse responses (CIR) from different transmit antennas. The proposed approach consists of three stages at each receive antenna. First, coarse timing and frequency offset estimates are obtained. Then, an advanced timing, relative timing indices, and the corresponding CIR estimates at the second stage are obtained using the generalized ML estimation based on a sliding observation vector. Finally, the fine time adjustment based on the minimum mean squared error criterion is performed. From the simulation results, the proposed approach has excellent performance in timing synchronization under several channel models at signal-to-noise ratio smaller than $1 \mathrm{~dB}$.

Keywords: Timing synchronization; Channel estimation; Multiple-input single-output (MISO); Multiple-input multiple-output (MIMO); Orthogonal frequency division multiplexing (OFDM); Generalized maximum-likelihood (ML) estimation; Training sequences
\end{abstract}

\section{Introduction}

In communications systems, timing and channel estimates are two important parameters at the receiver in order to reconstruct the signal. To further enhance the system performance, spatial diversity techniques can be applied to communications systems. By employing the spatial diversity techniques, multiple transmit antenna arrays are known to perform better than the conventional single transmit antenna because the effects of multipath fading channels and interference can be more effectively exploited [1,2]. Multiple-input single-output (MISO) and multiple-input multiple-output (MIMO) use multiple transmit antenna arrays and offer significant increases in data throughput and link range without additional bandwidth or increased transmit power. Recently, in wireless local area networks, orthogonal frequency division multiplexing (OFDM) has been of great interest

*Correspondence: kungx012@umn.edu

Department of Electrical and Computer Engineering, University of Minnesota, Minneapolis, MN 55455, USA for both wired and wireless applications due to its high spectrum efficiency and robustness against multipath fading channels [3], and the resultant MISO-OFDM and MIMO-OFDM systems have been recognized as promising solutions to support next generation broadband wireless communications systems [4]. However, compared with the single carrier systems, OFDM systems are much more sensitive to synchronization errors [5]. Several approaches have been proposed to address this problem [6-14]. In addition, the performance of multiple-input signal processing depends on the amount of channel information. Therefore, excellent synchronization and channel estimation are essential to fully exploit the advantages of MISO-OFDM and MIMO-OFDM systems.

Various techniques based on training sequences have been proposed to obtain accurate synchronization and channel estimation [9-19]. In [15], a training sequence arrangement based on modulated orthogonal sequences (MOS) for MIMO-OFDM systems was proposed to synchronize different transmit antennas. However, the

\section{Springer}

(c) 2013 Kung and Parhi; licensee Springer. This is an Open Access article distributed under the terms of the Creative Commons Attribution License (http://creativecommons.org/licenses/by/2.0), which permits unrestricted use, distribution, and reproduction in any medium, provided the original work is properly cited. 
mutual interference among training sequences transmitted by different transmit antennas becomes more severe when the number of transmit antennas increases, and the synchronization performance degrades correspondingly. In [16], a joint fine time synchronization and channel estimation scheme based on a nonoverlapping training sequence arrangement was proposed. Although the proposed training sequence arrangement is free from mutual interference, the time-division alignment of training sequence decreases the system efficiency. In [17], a joint fine time synchronization and channel estimation approach based on a unique MOS was proposed. The mutual interference among different training sequences is eliminated because of this careful training sequence arrangement. This joint approach relies on a threshold to identify the first significant tap using the estimated channel impulse responses (CIR). However, the threshold has to be determined in a trial-and-error manner. In [18], a joint timing synchronization and channel estimation approach based on the same training sequence arrangement was proposed. First, the optimal and suboptimal threshold factors are derived by analyzing the probability density functions of the cross-correlation function outputs in Rayleigh and Ricean fading channels. Then, a majority vote refinement approach which requires multiple timing estimates was also proposed for fine time adjustment in MIMO systems. However, these derived optimal and suboptimal threshold factors could be determined only when signal-to-noise ratio (SNR) exceeds $0 \mathrm{~dB}$, and this joint approach is not suitable for MISO systems.

This paper develops a joint timing synchronization and channel estimation algorithm suitable for downlink MIMO networks based on a time-domain training sequence arrangement in the short-range wireless transmission environment. Unlike the previous works [9-13,17,18], this paper not only introduces the use of sliding observation vector in timing synchronization phase but also applies the minimum mean squared error (MMSE) criterion to perform fine time adjustment. In this paper, we first obtain a coarse timing offset using the cross-correlation function outputs based on the proposed training sequences at each receive antenna and then apply the generalized maximum-likelihood (ML) algorithm to find the advanced timing, relative timing indices, and the corresponding CIR estimates. The reason why we use the advanced timing instead of coarse timing offset to perform fine time adjustment is that we can have better timing synchronization performance at very low SNR. Finally, the designed metric based on the MMSE criterion is utilized to perform fine time adjustment. Simulation results verify the effectiveness of our proposed algorithm. This paper is an extended version of [19]. This paper addresses synchronization and channel estimation aspects of a multiple transmit antenna system, whereas
[19] only considered those of a single transmit antenna system.

This paper is organized as follows. Section 2 describes the system model. In Section 3, the proposed joint fine time synchronization and channel estimation scheme is presented. Simulation results are provided in Section 4 . Finally, Section 5 concludes this paper.

\section{System description}

In this paper, we consider a centralized multiple-input OFDM-based communications system, which means that the transmit antennas are co-located on a single device. Moreover, receive antennas are not required to be colocated on a single device. Let us consider a multiple-input system that is formed by $N_{T}$ transmit antennas and $N_{R}$ receive antennas, where $N_{T}>1$ and $N_{R} \geq 1$. The training sequence arrangement used to assist joint timing synchronization and channel estimation is shown in Figure 1, where the training sequence assigned to each transmit antenna is composed of two identical pseudo-noise (PN) sequences and a guard interval. The reason why we utilize the PN sequences in the proposed training sequence arrangement is that sequences are easily generated to specify different transmit antennas. Let $\mathbf{p}_{\alpha_{1}}^{T}=p_{\alpha_{1}}(n)$ and $\mathbf{p}_{\alpha_{2}}^{T}=p_{\alpha_{2}}(n)$ denote training sequences assigned to the $\alpha_{1}$ th transmit antenna and the $\alpha_{2}$ th transmit antenna, respectively, where $\mathbf{p}_{\alpha_{1}}^{T}=\left[\mathbf{c}_{\alpha_{1}}^{T} \mathbf{g}^{T} \mathbf{c}_{\alpha_{1}}^{T}\right], \mathbf{p}_{\alpha_{2}}^{T}=\left[\begin{array}{ll}\mathbf{c}_{\alpha_{2}}^{T} & \mathbf{g}^{T} \mathbf{c}_{\alpha_{2}}^{T}\end{array}\right]$, $n \in\left\{0,1, \ldots, 2 N_{c}+N_{g}-1\right\}, \mathbf{c}_{\alpha_{1}}^{T}$ and $\mathbf{c}_{\alpha_{2}}^{T}$ are the corresponding PN sequences, $\mathbf{g}^{T}$ is the guard interval, $N_{c}$ is the length of $\mathbf{c}_{\alpha_{1}}^{T}$ and $\mathbf{c}_{\alpha_{2}}^{T}, N_{g}$ is the length of $\mathbf{g}^{T}$, and $N_{g} \geq 0$. In order to eliminate the mutual interference from different transmit antennas, the cross-correlation values between different assigned PN sequences are zero, i.e.,

$$
\mathbf{c}_{\alpha_{1}}^{T} \cdot \mathbf{c}_{\alpha_{2}}=0, \forall \alpha_{1} \neq \alpha_{2}, \alpha_{1}, \alpha_{2} \in\left\{1,2, \ldots, N_{T}\right\} .
$$

Consider the transmitted packet at the $i$ th transmit antenna $\mathbf{s}_{i}^{T}=\left[\mathbf{p}_{i}^{T} \mathbf{x}_{i}^{T}\right]=\left\{s_{i}(n), \forall n \in \Omega\right\}$, where $\mathbf{x}_{i}^{T}$ consists of $m$ OFDM symbols, the length of $\mathbf{x}_{i}^{T}$ is $m \cdot\left(N+N_{C P}\right)$, $N$ represents the number of subcarriers in the OFDM system, $N_{C P}$ denotes the length of cyclic prefix, $m$ is a positive integer, and $\Omega=\left\{0,1, \cdots, m \cdot\left(N+N_{C P}\right)+2 N_{c}+N_{g}-1\right\}$. Assume cyclic prefix in each OFDM symbol and guard interval in $\mathbf{p}_{i}^{T}$ are longer than the maximum delay spread of the channel, and the path delays in the channels are sample-spaced. Therefore, the received signal at the $b$ th receiver can be expressed as follows:

$$
r_{b}(n)=e^{\frac{j 2 \pi \epsilon_{b}}{N}} \sum_{i=1}^{N_{T}} \sum_{k=0}^{K-1} h_{i b}(k) s_{i}\left(n-\tau_{b}-k\right)+w(n),
$$

where $b \in\left\{1, \ldots, N_{R}\right\}, \epsilon_{b}$ is the carrier frequency offset (CFO) normalized to the OFDM subcarrier spacing, $\tau_{b}$ is the timing offset from all transmit antennas to the $b$ th receive antenna, $h_{i b}(k)$ represents the $k$ th CIR from 

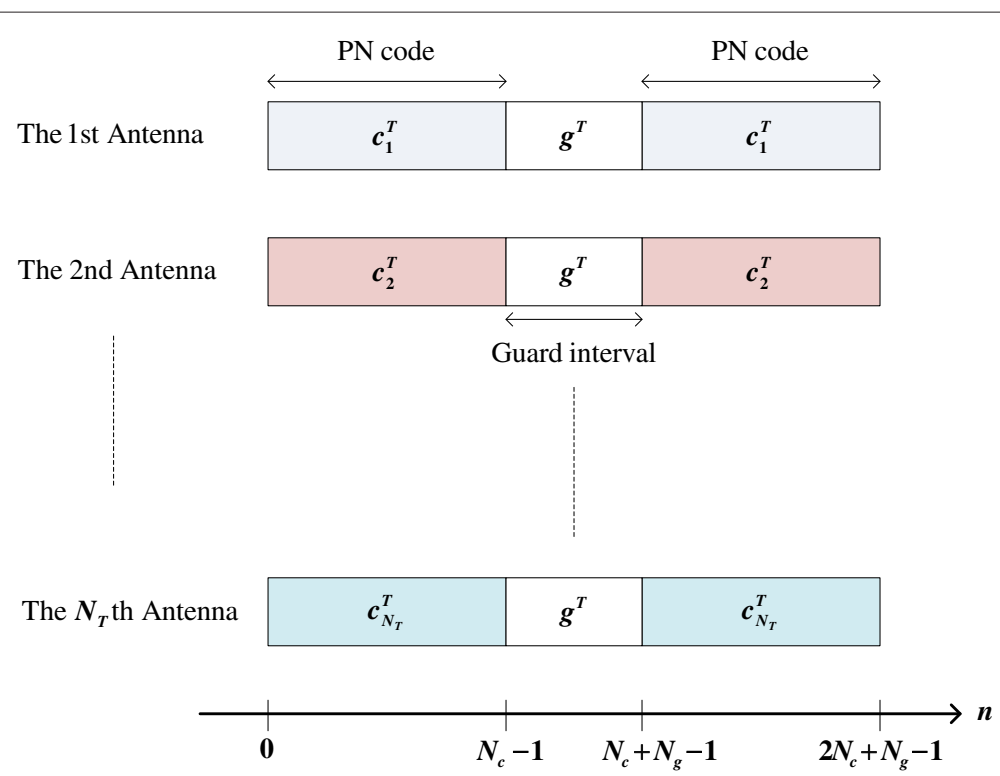

Figure 1 The proposed training sequence arrangement for centralized multiple-input systems.

the $i$ th transmitter to the $b$ th receiver, $K$ is the number of taps in the channel, and $w(n)$ is a complex additive white Gaussian noise (AWGN) sample. Based on the proposed training arrangement, if $N_{g} \geq K$, the training sequences are free of intersymbol interference caused by multipath fading channels. In addition, during each packet transmission, all channels are assumed to be slow time-varying channels, and the upper bounds for $2 N_{c}+N_{g}$ and $N_{g}$ are

$$
\begin{gathered}
T_{s} \cdot\left\{2 N_{c}+N_{g}+m \cdot\left(N+N_{C P}\right)\right\} \ll T_{c o h} \\
2 N_{c}+N_{g}<<\frac{T_{c o h}}{T_{s}}-m \cdot\left(N+N_{C P}\right) \\
N_{g}<<\frac{T_{c o h}}{T_{s}}-m \cdot\left(N+N_{C P}\right)-2 N_{c}
\end{gathered}
$$

where $T_{s}$ and $T_{c o h}$ represent the sample duration and the coherent time in channels, respectively. From Equation 5, the length of guard interval is bounded by

$$
K \leq N_{g}<<\frac{T_{c o h}}{T_{s}}-m \cdot\left(N+N_{C P}\right)-2 N_{c} .
$$

Therefore, the optimal bound for the length of training sequences, $\left\{\mathbf{p}_{i}^{T}, \forall i\right\}$, is

$$
2 N_{c}+K \leq 2 N_{c}+N_{g}<<\frac{T_{c o h}}{T_{s}}-m \cdot\left(N+N_{C P}\right) .
$$

\section{The proposed approach}

The state diagram of the proposed approach is shown in Figure 2. In Figure 2, the proposed approach consists of three blocks. In this section, we will thoroughly explain the mechanism in each block.

\subsection{Coarse timing and frequency synchronization}

It can be easily observed that the proposed training sequence is composed of two identical parts. Therefore, a coarse timing offset $(\mathrm{CTO})$ at the $b$ th receiver $\tau_{c, b}$ is obtained based on the cross-correlation function outputs as follows:

$$
\begin{aligned}
& \tau_{c, b}=\arg \max _{d \in \Omega_{d}}\left\{\left|M_{c, b}(d)\right|\right\} \\
& M_{c, b}(d)=\sum_{n=0}^{N_{c}-1} \frac{r_{b}^{*}(n+d) r_{b}\left(n+N_{c}+N_{g}+d\right)}{N_{c}},
\end{aligned}
$$

where $r_{b}^{*}(n)$ denotes the complex conjugate of $r_{b}(n)$, $\left|r_{b}(n)\right|$ represents the absolute value of $r_{b}(n), \Omega_{d}$ is the observation interval, $\Omega_{d}=\{0,1, \ldots, D-1\}, D$ is the length of observation interval, and $M_{c, b}(d)$ is the coarse timing metric at the $b$ th receiver. From Equations 2 and $8, M_{c, b}(d)$ will give a maximum value when $d$ is at the delayed timing of the line-of-sight (LOS) path in multipath fading channels. Then, the CFO $\hat{\epsilon}_{b}$ is

$$
\hat{\epsilon}_{b}=\frac{N}{2 \pi\left(N_{c}+N_{g}\right)} \tan ^{-1}\left(\frac{\Im\left\{M_{c, b}\left(\tau_{c, b}\right)\right\}}{\Re\left\{M_{c, b}\left(\tau_{c, b}\right)\right\}}\right),
$$

where $\Re\{x\}$ and $\mathfrak{\Im}\{x\}$ describe the real and the imaginary parts of $x$, respectively. After coarse frequency synchronization, the CFO-compensated received signal at the $b$ th receiver is 


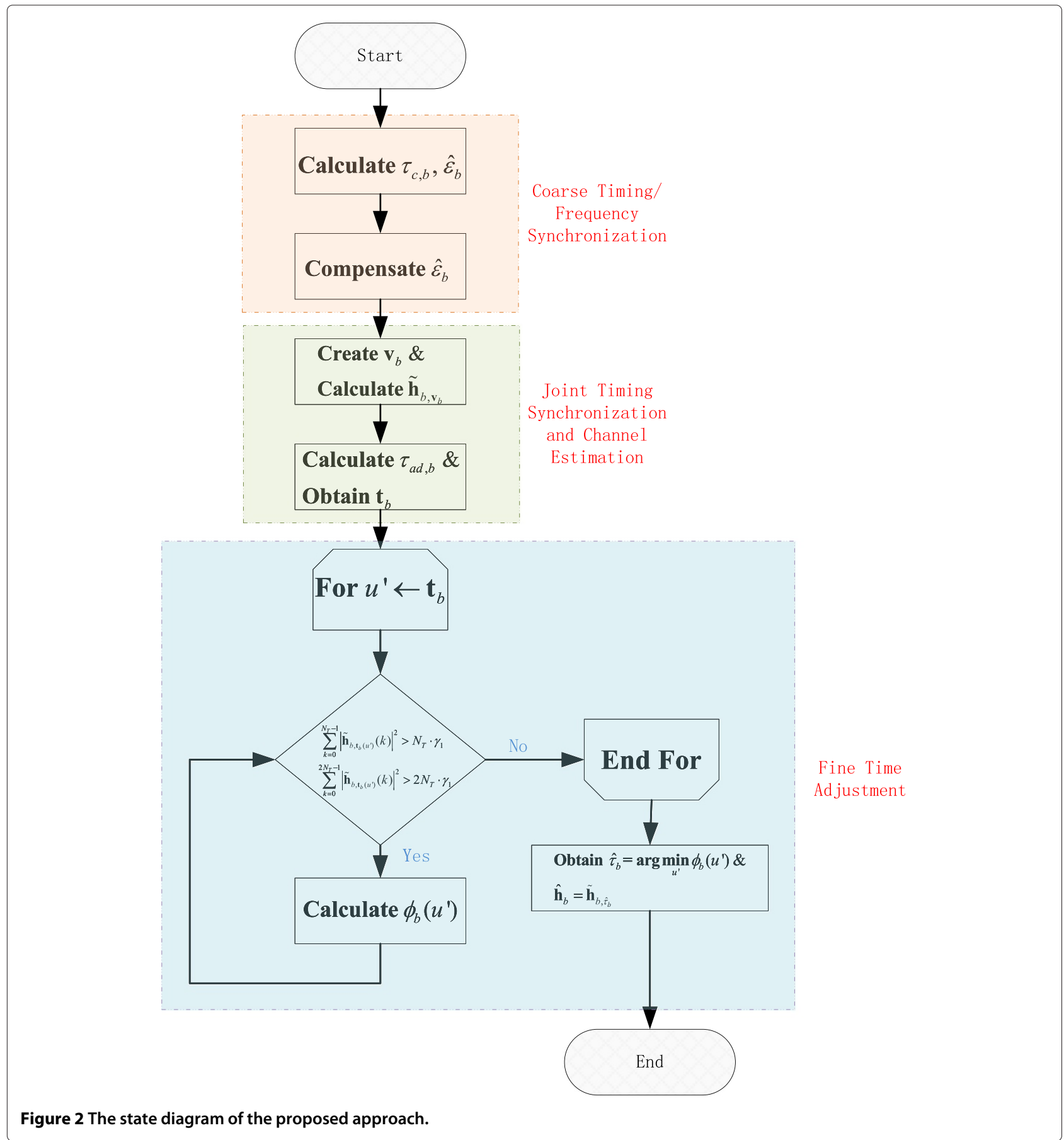

$$
\begin{aligned}
& \hat{r}_{b}(n) \\
& =r_{b}(n) \cdot e^{\frac{-j 2 \pi\left(\epsilon_{b}+\Delta \epsilon_{b}\right) n}{N}} \\
& =e^{\frac{-j 2 \pi\left(\Delta \epsilon_{b} n\right)}{N}} \sum_{i=1}^{N_{T}} \sum_{k=0}^{K-1} h_{i b}(k) s_{i}\left(n-\tau_{b}-k\right)+\hat{w}(n),
\end{aligned}
$$

\subsection{Generalized maximum-likelihood-based channel estimation}

(10) Assume there is no timing offset and CFO in Equation 2, the received training sequence at the $b$ th receiver can be expressed as follows:

$$
\mathbf{r}_{b}=\mathbf{S h}_{b}+\mathbf{w}
$$

where $\Delta \epsilon_{b}=\hat{\epsilon}_{b}-\epsilon_{b}$ denotes the residual CFO and $\hat{w}(n)=$ $w(n) e^{\frac{-j 2 \pi\left(\epsilon_{b}+\Delta \epsilon_{b}\right) n}{N}}$.

where

$$
\mathbf{r}_{b}=\left[r_{b}(0) r_{b}(1) \cdots r_{b}\left(N^{\prime}-1\right)\right]^{T},
$$




$$
\mathbf{S}=\left(\begin{array}{cccccccccc}
p_{1}(0) & \cdots & p_{N_{T}}(0) & 0 & \cdots & 0 & \cdots & 0_{(K-1) \times 1} & \cdots & 0_{(K-1) \times 1} \\
p_{1}(1) & \cdots & p_{N_{T}}(1) & p_{1}(0) & \cdots & p_{N_{T}}(0) & \ddots & p_{1}(0) & \cdots & p_{N_{T}}(0) \\
\vdots & \vdots & \vdots & \vdots & \vdots & \vdots & \ddots & \vdots & \vdots & \vdots \\
p_{1}\left(N^{\prime}-1\right) & \cdots & p_{N_{T}}\left(N^{\prime}-1\right) & p_{N_{T}}\left(N^{\prime}-2\right) & \cdots & p_{N_{T}}\left(N^{\prime}-2\right) & \cdots & p_{1}\left(N^{\prime}-K\right) & \cdots & p_{N_{T}}\left(N^{\prime}-K\right)
\end{array}\right),
$$

$$
\begin{aligned}
\mathbf{h}_{b}= & {\left[h_{1 b}(0) \cdots h_{N_{T} b}(0) h_{1 b}(1) \cdots h_{N_{T} b}(1) \cdots\right.} \\
& \left.h_{1 b}(K-1) \cdots h_{N_{T} b}(K-1)\right]^{T}, \\
\mathbf{w}= & {\left[w(0) w(1) \cdots w\left(N^{\prime}-1\right)\right]^{T}, }
\end{aligned}
$$

$N^{\prime}=2 N_{c}+N_{g}, \mathbf{w} \sim N\left(0_{N^{\prime} \times 1}, \sigma_{w}^{2} \mathbf{I}_{N^{\prime} \times N^{\prime}}\right), \mathbf{I}_{N^{\prime} \times N^{\prime}}$ is a $N^{\prime} \times N^{\prime}$ identity matrix, $\{w(n), \forall n\}$ is independent identically distributed, and $h_{i b}(k)$ is the $k$ th tap CIR from the $i$ th transmit antenna to the $b$ th receive antenna. In Equation 14, $\mathbf{h}_{b}$ contains different CIRs from all transmit antennas. In general, the received training sequence is

$$
\mathbf{r}_{b}(\tau)=\left[r_{b}(\tau) r_{b}(\tau+1) \cdots r_{b}\left(\tau+N^{\prime}-1\right)\right]^{T},
$$

where Equation 12 is a special case of Equation 16 when $\tau=0$ and $\epsilon_{b}=0$. Then, the likelihood function is given by

$$
\Lambda\left(\mathbf{h}_{b}\right)=\frac{1}{\left(\pi \sigma_{w}^{2}\right)^{N^{\prime}}} \exp \left\{\frac{-1}{\sigma_{w}^{2}}\left\|\mathbf{r}_{b}(\tau)-\Gamma\left(\epsilon_{b}\right) \mathbf{S h}_{b}\right\|^{2}\right\}
$$

and the ML estimate of $\mathbf{h}_{b}$ can be obtained by

$$
\begin{aligned}
& \hat{\mathbf{h}}_{b, \mathrm{ML}}=\arg \max _{\mathbf{h}_{b}} \Lambda\left(\mathbf{h}_{b}\right) \\
& =\left(\mathbf{S}^{H} \mathbf{S}\right)^{-} \mathbf{S}^{H} \boldsymbol{\Gamma}^{H}\left(\epsilon_{b}\right) \mathbf{r}_{b}(\tau) \\
& =\mathbf{S}^{\dagger} \boldsymbol{\Gamma}^{H}\left(\epsilon_{b}\right) \mathbf{r}_{b}(\tau),
\end{aligned}
$$

where $\mathbf{A}^{H}$ is the Hermitian of $\mathbf{A}, \mathbf{B}^{-}$is the generalized inverse of $\mathbf{B}, \mathbf{S}^{\dagger}$ is the Moore-Penrose pseudo-inverse of $\mathbf{S}$,

$$
\boldsymbol{\Gamma}\left(\epsilon_{b}\right)=\operatorname{diag}\left(e^{\frac{j 2 \pi \epsilon_{b} \tau}{N}}, e^{\frac{j 2 \pi \epsilon_{b}(\tau+1)}{N}}, \cdots, e^{\frac{j 2 \pi \epsilon_{b}\left(\tau+N^{\prime}-1\right)}{N}}\right)
$$

and $\operatorname{diag}(\cdot)$ represents a diagonal matrix. For fixed pseudo-sequences $\mathbf{c}_{i}, \forall i \in\left\{1, \ldots, N_{T}\right\}$, we only need to compute $\mathbf{S}^{\dagger}$ once, and different CIR estimates can be obtained simply by multiplying the received training sequence at each receiver with $\boldsymbol{S}^{\dagger}$.

\subsection{Joint timing synchronization and channel estimation} In this subsection, we utilize the coarse timing offset and CIR estimate based on the generalized ML criterion to develop a joint timing synchronization and channel estimation algorithm. After coarse timing synchronization, a sliding observation vector $\mathbf{v}_{b}$ at the $b$ th receiver is applied to obtain an advanced timing, relative timing indices, and the corresponding CIR estimates, where

$$
\begin{aligned}
\mathbf{v}_{b}^{T} & =\left[\tau_{c, b}+L \cdots \tau_{c, b}+1 \tau_{c, b} \tau_{c, b}-1 \cdots \tau_{c, b}-L\right] \\
& =\left\{v_{b}\left(l_{1}\right), \forall l_{1} \in \Omega_{v_{b}}\right\},
\end{aligned}
$$

$\Omega_{v_{b}}=\{0,1, \cdots, 2 L\}$, the length of observation interval is $2 L+1$, and $L$ is a positive integer without any constraint. In Equation 20, $\mathbf{v}_{b}$ consists of $2 L+1$ timing indices. Based on these timing indices in $\mathbf{v}_{b}$, the corresponding CIR estimates with $K^{\prime}$ taps are obtained by Equation 18, where

$$
\tilde{\mathbf{h}}_{b, v_{b}\left(l_{1}\right)}=\tilde{\mathbf{S}}^{\dagger} \hat{\mathbf{r}}_{b}\left(v_{b}\left(l_{1}\right)\right)
$$

$$
\begin{aligned}
\tilde{\mathbf{h}}_{b, v_{b}\left(l_{1}\right)}= & {\left[\tilde{h}_{1 b, v_{b}\left(l_{1}\right)}(0) \cdots \tilde{h}_{N_{T} b, v_{b}\left(l_{1}\right)}(0) \tilde{h}_{1 b, v_{b}\left(l_{1}\right)}(1) \cdots\right.} \\
& \tilde{h}_{N_{T} b, v_{b}\left(l_{1}\right)}(1) \cdots \tilde{h}_{1 b, v_{b}\left(l_{1}\right)}\left(K^{\prime}-1\right) \cdots \\
& \left.\tilde{h}_{N_{T} b, v_{b}\left(l_{1}\right)}\left(K^{\prime}-1\right)\right]^{T},
\end{aligned}
$$

$$
\tilde{\mathbf{S}}=\left(\begin{array}{cccccccccc}
p_{1}(0) & \cdots & p_{N_{T}}(0) & 0 & \cdots & 0 & \cdots & 0_{\left(K^{\prime}-1\right) \times 1} & \cdots & 0_{\left(K^{\prime}-1\right) \times 1} \\
p_{1}(1) & \cdots & p_{N_{T}}(1) & p_{1}(0) & \cdots & p_{N_{T}}(0) & \ddots & p_{1}(0) & \cdots & p_{N_{T}}(0) \\
\vdots & \vdots & \vdots & \vdots & \vdots & \vdots & \ddots & \vdots & \vdots & \vdots \\
p_{1}\left(N^{\prime}-1\right) & \cdots & p_{N_{T}}\left(N^{\prime}-1\right) & p_{N_{T}}\left(N^{\prime}-2\right) & \cdots & p_{N_{T}}\left(N^{\prime}-2\right) & \cdots & p_{1}\left(N^{\prime}-K^{\prime}\right) & \cdots & p_{N_{T}}\left(N^{\prime}-K^{\prime}\right)
\end{array}\right),
$$




$$
\begin{aligned}
& \hat{\mathbf{r}}_{b}\left(v_{b}\left(l_{1}\right)\right)= \\
& {\left[\hat{r}_{b}\left(v_{b}\left(l_{1}\right)\right) \hat{r}_{b}\left(v_{b}\left(l_{1}\right)+1\right) \cdots \hat{r}_{b}\left(v_{b}\left(l_{1}\right)+N^{\prime}-1\right)\right]^{T},}
\end{aligned}
$$

$\forall l_{1} \in \Omega_{v_{b}}, \tilde{\mathbf{h}}_{b, v_{b}\left(l_{1}\right)}$ is the CIR estimate corresponding to the time index $v_{b}\left(l_{1}\right)$, and $\tilde{h}_{i b, v_{b}\left(l_{1}\right)}(k)$ is the estimated $(k+1)$ th tap CIR from the $i$ th transmit antenna to the $b$ th receive antenna corresponding to the time index $v_{b}\left(l_{1}\right)$. In order to avoid any loss of the channel information, $K^{\prime}$ should be at least equal to or greater than $K$.

If $N_{g} \geq K, \tilde{\mathbf{h}}_{b, v_{b}\left(l_{1}\right)}$ can be obtained in an efficient way by averaging two channel estimates, $\overline{\mathbf{h}}_{A, v_{b}\left(l_{1}\right)}$ and $\overline{\mathbf{h}}_{B, v_{b}\left(l_{1}\right)}$, where

$$
\begin{aligned}
\overline{\mathbf{h}}_{A, v_{b}\left(l_{1}\right)} & =\overline{\mathbf{S}}^{\dagger} \overline{\mathbf{r}}_{b}\left(v_{b}\left(l_{1}\right)\right), \\
\overline{\mathbf{h}}_{B, v_{b}\left(l_{1}\right)} & =\overline{\mathbf{S}}^{\dagger} \overline{\mathbf{r}}_{b}\left(v_{b}\left(l_{1}\right)+N_{c}+N_{g}\right), \\
\tilde{\mathbf{h}}_{b, v_{b}\left(l_{1}\right)} & =\frac{\overline{\mathbf{h}}_{A, v_{b}\left(l_{1}\right)}+\overline{\mathbf{h}}_{B, v_{b}\left(l_{1}\right)}}{2},
\end{aligned}
$$

$$
\begin{aligned}
& \overline{\mathbf{r}}_{b}\left(v_{b}\left(l_{1}\right)\right)= \\
& {\left[\hat{r}_{b}\left(v_{b}\left(l_{1}\right)\right) \hat{r}_{b}\left(v_{b}\left(l_{1}\right)+1\right) \cdots \hat{r}_{b}\left(v_{b}\left(l_{1}\right)+N_{c}-1\right)\right]^{T},}
\end{aligned}
$$

$$
\begin{aligned}
& \overline{\mathbf{r}}_{b}\left(v_{b}\left(l_{1}\right)+N_{c}+N_{g}\right)= \\
& {\left[\hat{r}_{b}\left(v_{b}\left(l_{1}\right)+N_{c}+N_{g}\right) \hat{r}_{b}\left(v_{b}\left(l_{1}\right)+N_{c}+N_{g}+1\right) \cdots\right.} \\
& \left.\quad \hat{r}_{b}\left(v_{b}\left(l_{1}\right)+N^{\prime}-1\right)\right]^{T},
\end{aligned}
$$

and

$$
\overline{\mathbf{S}}=\left(\begin{array}{cccccccccc}
p_{1}(0) & \cdots & p_{N_{T}}(0) & 0 & \cdots & 0 & \cdots & 0_{\left(K^{\prime}-1\right) \times 1} & \cdots & 0_{\left(K^{\prime}-1\right) \times 1} \\
p_{1}(1) & \cdots & p_{N_{T}}(1) & p_{1}(0) & \cdots & p_{N_{T}}(0) & \ddots & p_{1}(0) & \cdots & p_{N_{T}}(0) \\
\vdots & \vdots & \vdots & \vdots & \vdots & \vdots & \ddots & \vdots & \vdots & \vdots \\
p_{1}\left(N_{c}-1\right) & \cdots & p_{N_{T}}\left(N_{c}-1\right) & p_{N_{T}}\left(N_{c}-2\right) & \cdots & p_{N_{T}}\left(N_{c}-2\right) & \cdots & p_{1}\left(N_{c}-K^{\prime}\right) & \cdots & p_{N_{T}}\left(N_{c}-K^{\prime}\right)
\end{array}\right) .
$$

After we obtain $2 L+1$ CIR estimates, the advanced timing at the $b$ th receiver $\tau_{a d, b}$ is given by

$$
\tau_{a d, b}=\arg \max _{v_{b}\left(l_{1}\right), l_{1} \in \Omega_{v_{b}}} \sum_{k=0}^{2 N_{T}-1}\left|\tilde{\mathbf{h}}_{b, v_{b}\left(l_{1}\right)}(k)\right|,
$$

where $\tilde{\mathbf{h}}_{b, v_{b}\left(l_{1}\right)}(0)$ is the first tap in Equation 22. Then, relative timing indices in the modified sliding observation vector $\mathbf{t}_{b}^{T}$ and the corresponding CIR estimates $\tilde{\mathbf{h}}_{b, t_{b}\left(l_{2}\right)}$ are fed forward to perform fine time adjustment, where

$$
\mathbf{t}_{b}^{T}=\left[\tau_{a d, b} \tau_{a d, b}-1 \cdots \tau_{c, b}-L\right]=\left\{t_{b}\left(l_{2}\right), l_{2} \in \Omega_{t_{b}}\right\},
$$

and $\Omega_{t_{b}}=\left\{0,1, \cdots, \tau_{a d, b}-\tau_{c, b}+L\right\}$.

\subsection{Fine time adjustment}

In this subsection, we exploit the MMSE criterion to perform fine time adjustment in an iterative manner based on the information of relative timing indices and the corresponding CIR estimates. First, two thresholds on the sum of the first $N_{T}$ tap powers $N_{T} \cdot \gamma_{1}$ and the sum of the first $2 N_{T}$ tap powers $2 N_{T} \cdot \gamma_{1}$ are chosen in order to eliminate the AWGN effect and to reduce the computational complexity, where $\gamma_{1}$ should be less than the tap power of the first path in the channel model. In other words, if $\sum_{k=0}^{N_{T}-1}\left|\tilde{\mathbf{h}}_{b, \tau_{a d, b}-1}(k)\right|^{2}<$ $N_{T} \cdot \gamma_{1}$ or $\sum_{k=0}^{2 N_{T}-1}\left|\tilde{\mathbf{h}}_{b, \tau_{a d, b}-1}(k)\right|^{2}<2 N_{T} \cdot \gamma_{1}, \tau_{a d, b}$ is the estimated timing offset; otherwise, the algorithm keeps running until $\sum_{k=0}^{N_{T}-1}\left|\tilde{\mathbf{h}}_{b, t_{b}(f)}(k)\right|^{2}<N_{T}$. $\gamma_{1}$ or $\sum_{k=0}^{2 N_{T}-1}\left|\tilde{\mathbf{h}}_{b, t_{b}(f)}(k)\right|^{2}<2 N_{T} \cdot \gamma_{1}$ for some $f$, where $f \in \Omega_{t_{b}}$.

Let $\mathbf{q}_{t_{b}\left(l_{2}\right)}^{T}$ denote the convolution of the training sequence and the corresponding CIR estimate $\tilde{\mathbf{h}}_{b, t_{b}\left(l_{2}\right)}^{T}$, where

$$
\mathbf{q}_{t_{b}\left(l_{2}\right)}^{T}=\sum_{i=1}^{N_{T}} \mathbf{p}_{i}^{T} * \tilde{\mathbf{h}}_{i b, t_{b}\left(l_{2}\right)}^{T},
$$

$$
\tilde{\mathbf{h}}_{i b, t_{b}\left(l_{2}\right)}^{T}=\left[\tilde{h}_{i b, t_{b}\left(l_{2}\right)}(0) \tilde{h}_{i b, t_{b}\left(l_{2}\right)}(1) \cdots \tilde{h}_{i b, t_{b}\left(l_{2}\right)}\left(K^{\prime}-1\right)\right],
$$


and $\tilde{\mathbf{h}}_{i b, t_{b}\left(l_{2}\right)}^{T}$ is the CIR estimate from the $i$ th transmit antenna to the $b$ th receive antenna. Then, the mean squared error (MSE) of the timing index $t_{b}\left(l_{2}\right)$ is given by

$$
\phi_{b}\left(t_{b}\left(l_{2}\right)\right)=\sum_{n=0}^{2 N_{c}+N_{g}+K^{\prime}-1}\left|\hat{\mathbf{r}}_{b}\left(t_{b}\left(l_{2}\right)+n\right)-\mathbf{q}_{t_{b}\left(l_{2}\right)}(n)\right|^{2} .
$$

Thus, the estimated timing offset can be obtained by solving

$$
\begin{aligned}
\min & \phi_{b}\left(t_{b}\left(l_{2}\right)\right) \\
\text { s.t. } & \sum_{k=0}^{N_{T}-1}\left|\tilde{\mathbf{h}}_{b, t_{b}\left(u^{\prime}\right)}(k)\right|^{2}>N_{T} \cdot \gamma_{1}, \\
& \sum_{k=0}^{2 N_{T}-1}\left|\tilde{\mathbf{h}}_{b, t_{b}\left(u^{\prime}\right)}(k)\right|^{2}>2 N_{T} \cdot \gamma_{1} .
\end{aligned}
$$

From Equations 31 and 33 and reasonable CIR estimates, we have

$$
\phi_{b}\left(\tau_{b}+\delta\right)>\phi_{b}\left(\tau_{b}\right),
$$

where $\delta \neq 0, \delta$ is an integer, and $\tau_{b}+\delta \in \mathbf{t}_{b}^{T}$. Consider a set $\Omega_{U}=\{0,1, \cdots, u-1\}$ composed of consecutive timing indices that satisfy the following conditions

$$
\begin{cases}\sum_{k=0}^{N_{T}-1} \quad\left|\tilde{\mathbf{h}}_{b, t_{b}\left(u^{\prime}\right)}(k)\right|^{2}>N_{T} \cdot \gamma_{1}, \\ \sum_{k=0}^{2 N_{T}-1}\left|\tilde{\mathbf{h}}_{b, t_{b}\left(u^{\prime}\right)}(k)\right|^{2}>2 N_{T} \cdot \gamma_{1}, \\ \forall u^{\prime} \in \Omega_{U} .\end{cases}
$$

Then, the estimated timing offset $\hat{\tau}_{b}$ and the CIR estimate $\hat{\mathbf{h}}_{b}$ at the $b$ th receiver based on the thresholds and Equation 33 can be expressed as follows:

$$
\left\{\begin{array}{l}
\hat{\tau}_{b}=\arg \min _{t_{b}\left(u^{\prime}\right), u^{\prime} \in \Omega_{U}} \phi_{b}\left(t_{b}\left(u^{\prime}\right)\right) \\
\hat{\mathbf{h}}_{b}=\tilde{\mathbf{h}}_{b, \hat{\tau}_{b}} .
\end{array}\right.
$$

The detailed procedure of fine time adjustment is described in Algorithm 1.

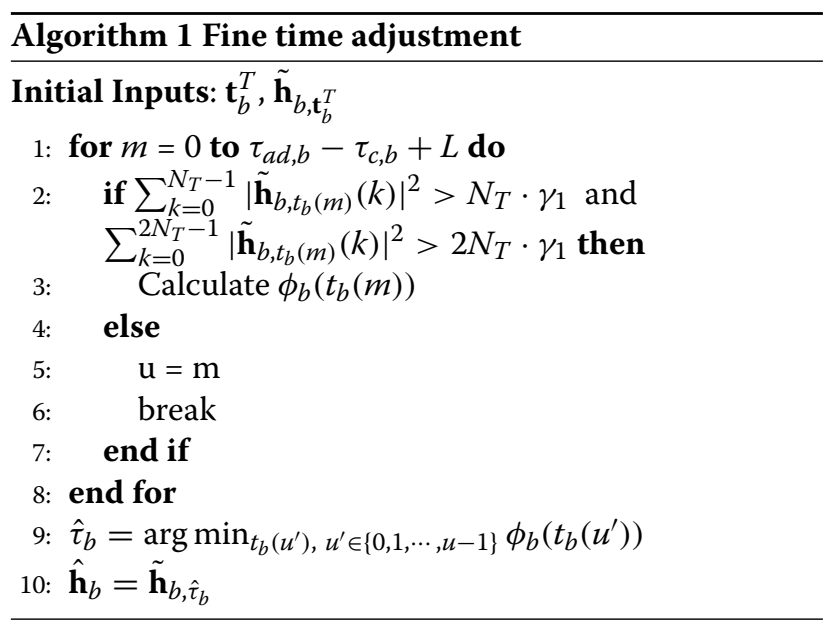

\section{Simulation results}

Packet-based $N_{T} \times N_{R}$ MIMO-OFDM systems were used for simulations, where each packet consists of a training sequence followed by 34 random OFDM data symbols. In this paper, we mainly consider $2 \times 2$ and $3 \times 3$ MIMOOFDM systems, where $N_{T} \times N_{R}=2 \times 2$ and $N_{T} \times$ $N_{R}=3 \times 3$. Gold code is selected to generate the pseudonoise sequences used in the proposed training sequence arrangement with the spreading factor equal to 1 . The training sequence of each packet is 80 identical samples at each transmitter, where $N_{c}=32$ and $N_{g}=16$. The structure of OFDM data symbols follows the IEEE 802.11a standard defined in [20], where $N=64$ and $N_{C P}=16$. Quaternary phase-shift keying modulation was adopted in simulations.

The power profiles of different channel models used for performance analysis and simulation are summarized in Table 1 , where $\sigma_{i}^{2}$ represents the $i+1$ th tap power in the corresponding channel. Different fading channels (Rayleigh, Ricean, and Nakagami) would not affect the results because tap powers in the channel models have been defined in Table 1. Channel models I and II (CH I and $\mathrm{CH}$ II) represent multipath fading channels with nonline-of-sight (NLOS) propagation, and channel model III (CH III) is a typical multipath fading channel with LOS propagation. For $\mathrm{CH}$ I, the power of second tap dominates all channel taps. As for $\mathrm{CH}$ II, the third tap has the strongest power in the channel, and it is the worst channel model to evaluate the performance of timing synchronization in this paper. Moreover, all channels are assumed to be quasi-stationary during each packet transmission. In this paper, all transmit-receive links follow the same channel model.

The main motivation of this paper is to achieve perfect timing synchronization in very low SNR environments. The parameters $L$ and $K^{\prime}$ related to the observation interval in joint timing synchronization and channel estimation are set to be 50 and 6 , respectively. Therefore, the number of possible relative timing indices for fine time adjustment in our proposed approach is approximately equal to 50. For comparison, the designed maximum channel delay spread for fine time adjustment used in [17] is set to 20 samples. In addition, the coarse timing estimate in the proposed approach is provided to [17] before the

Table 1 The power profiles of different channel models

\begin{tabular}{cccc}
\hline & CH I (NLOS) & CH II (NLOS) & CH III (LOS) \\
\hline$\sigma_{0}^{2}$ & 0.1232 & 0.1285 & 0.7211 \\
$\sigma_{1}^{2}$ & 0.7711 & 0.2223 & 0.2338 \\
$\sigma_{2}^{2}$ & 0.1029 & 0.6000 & 0.0420 \\
$\sigma_{3}^{2}$ & 0.0015 & 0.0478 & 0.0022 \\
$\sigma_{4}^{2}$ & 0.0001 & 0.0010 & 0.0006 \\
$\sigma_{5}^{2}$ & 0.0002 & 0.0001 & 0.0001 \\
\hline
\end{tabular}




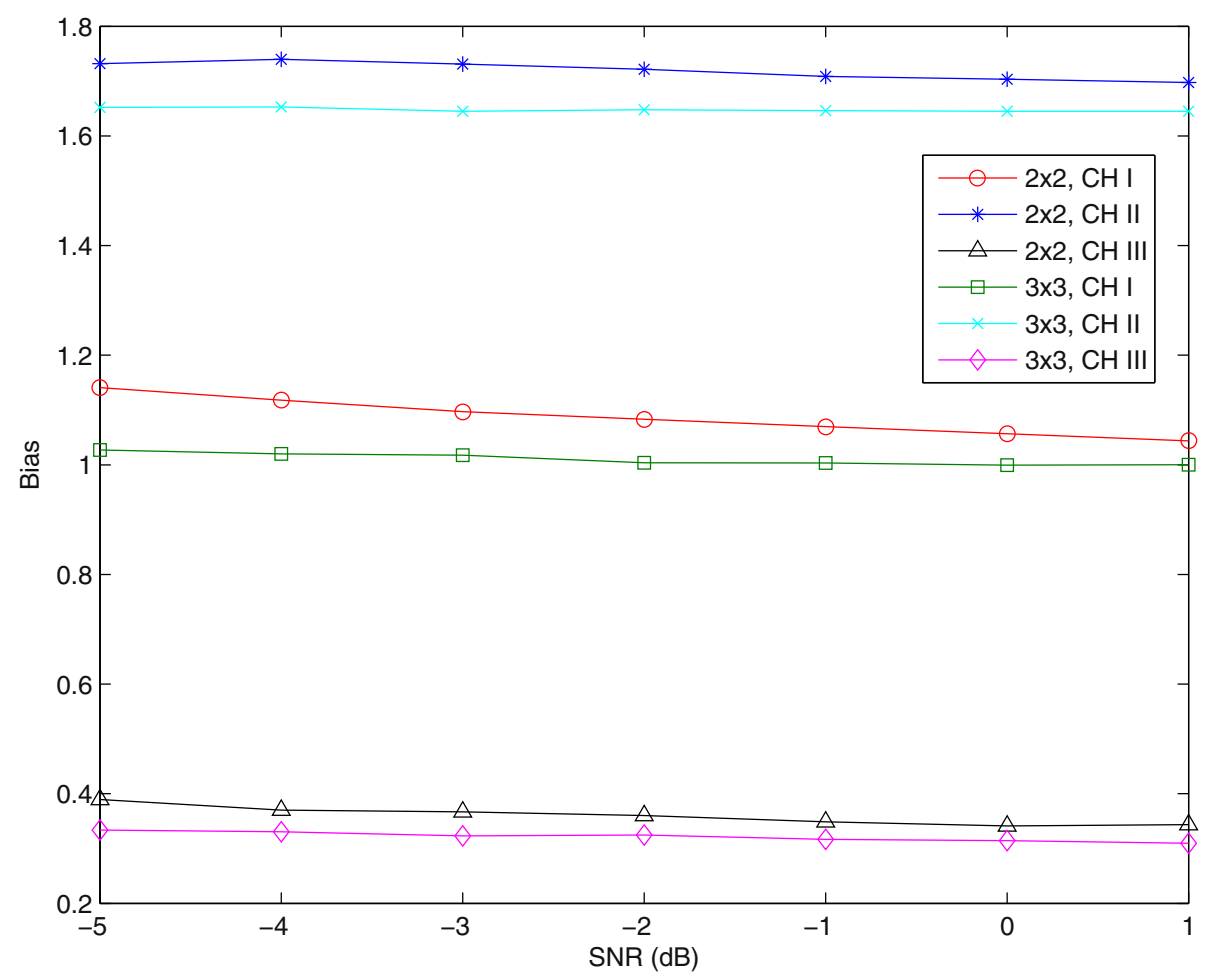

Figure 3 The averaged bias of coarse timing estimates, $E\left[\hat{\tau}_{c}-\tau\right]$, where $E[\cdot]$ is the expectation function.

proposed joint timing synchronization and channel estimation scheme is performed.

Figure 3 shows the averaged bias of CTO using our proposed algorithm at each receive antenna. In Figure 3, the averaged biases of $\mathrm{CTO}$ in $\mathrm{CH}$ I and $\mathrm{CH}$ II are greater than 1, and the averaged biases of CTO in $\mathrm{CH}$ III are approximately equal to 0 . The simulation results of estimated CTO show that the proposed algorithm provides good initial values for further processing of timing synchronization. In addition, constant CFOs $\left(\epsilon_{b}\right)$ equal to 0.5 are introduced to demonstrate the performance of CFO estimator. The mean square errors of estimated CFOs $\left(\left|\hat{\epsilon}_{b}-\epsilon_{b}\right|^{2}\right)$ in all channel models are listed in Table 2. From Table 2 , it is indicated that the residual CFO $\left(\Delta \epsilon_{b}=\right.$ $\left.\hat{\epsilon}_{b}-\epsilon_{b}\right)$ can be modeled as a random variable that is uniformly distributed within $[-0.04,0.04]$. Figure 3 and Table 2 demonstrate that our proposed algorithm has excellent performance in coarse timing and frequency synchronization.

After we discuss the performance in CFO and CTO estimators, the performance of timing synchronization is evaluated with a residual CFO $\Delta \epsilon_{b}=0.1$. The threshold $\gamma_{1}$ defined in fine time adjustment is 0.05 . We demonstrate the performance of timing synchronization using the probability of perfect timing synchronization, the bias of the time estimator, and the root mean squared error (RMSE) of the time estimator. The corresponding results are reported in Figures 4, 5, and 6. The perfect timing synchronization is defined as the successful acquisition of the position of the first tap in channel models. The actual threshold used in [17] is $\alpha\left|\hat{h}_{\max }\right|$, where $\alpha$ is a threshold factor and $\left|\hat{h}_{\max }\right|$ is the strongest channel tap gain estimate. In this paper, pre-simulations and mathematical derivations are not required to choose thresholds for fine time adjustment $[9,10,12,13]$. Moreover, the performance of timing synchronization is calculated based on the estimated timing offset at each receive antenna. In Figure 4, simulation results show that our proposed approach has better performance in timing synchronization at very low SNR. In $\mathrm{CH} \mathrm{I,} \mathrm{CH} \mathrm{II,} \mathrm{and} \mathrm{CH}$ III, the proposed approach achieves $99 \%$ in the probability of perfect timing synchronization when SNR exceeds -3dB. As

Table 2 The mean squared error of estimated coarse frequency offset at each receive antenna

\begin{tabular}{lccccc}
\hline & SNR $=-\mathbf{5 d B}$ & SNR $=-\mathbf{3 d B}$ & SNR $=-\mathbf{1 d B}$ & SNR $=\mathbf{0 d B}$ & SNR $=1 \mathbf{d B}$ \\
\hline Proposed $(2 \times 2 \mathrm{MIMO})$ & 0.0011 & 0.0006 & 0.0004 & 0.0003 & 0.0002 \\
Proposed $(3 \times 3 \mathrm{MIMO})$ & 0.0012 & 0.0007 & 0.0004 & 0.0003 & 0.0003 \\
\hline
\end{tabular}




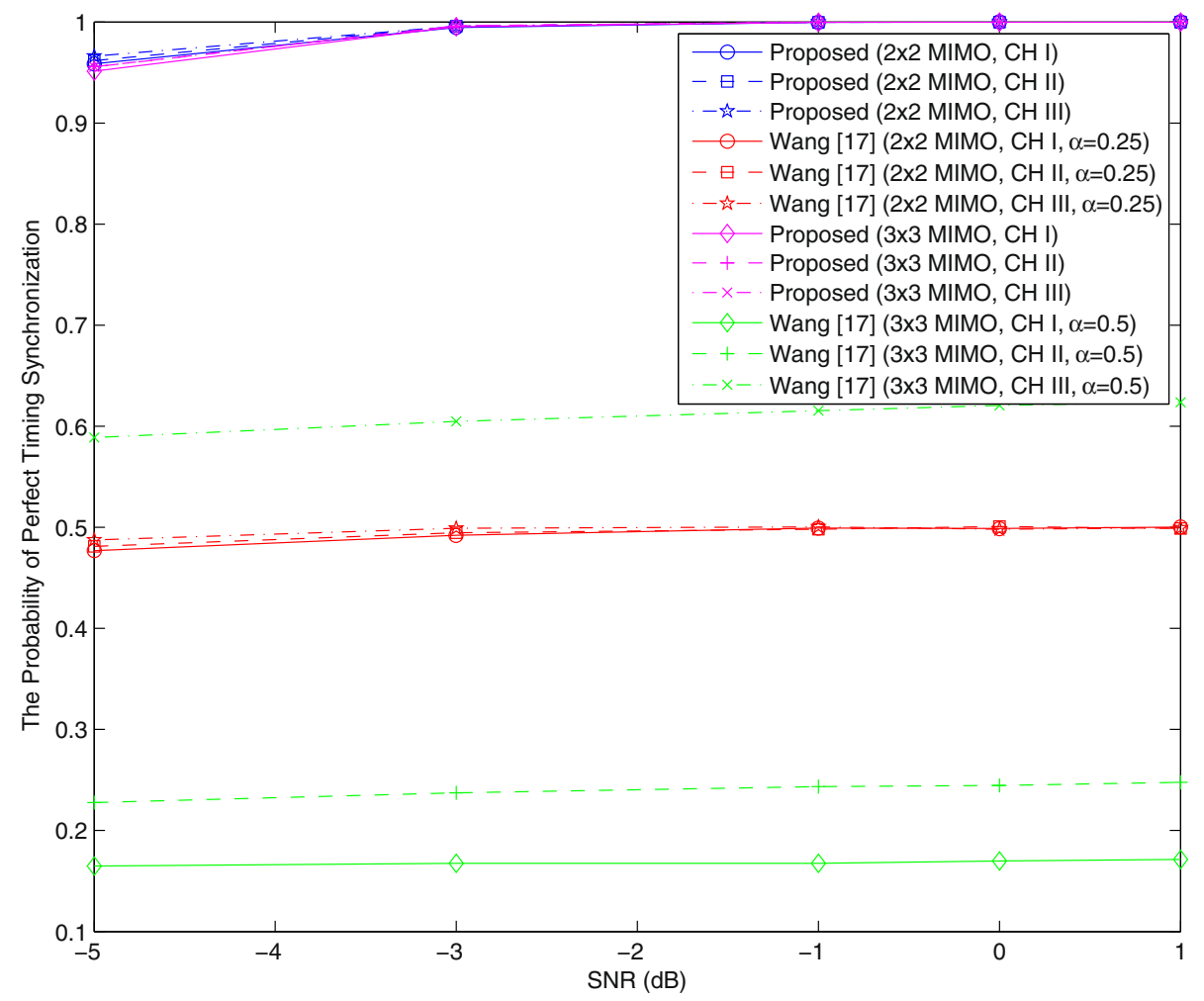

Figure 4 The probability of perfect timing synchronization, $\operatorname{prob}(\tau=\hat{\tau})$, where $\operatorname{prob}(\cdot)$ is the probability function and $\hat{\tau}$ is the estimated timing offset.

for [17], different choices in threshold factor $\alpha$ may lead to the poor performance in timing synchronization. In Figure 4, the maximum probability of perfect timing synchronization in [17] is less than 0.624 in CH III, where $\alpha=$ 0.5 . However, in this case, the probabilities of perfect timing synchronization are less than 0.25 in $\mathrm{CH}$ I and $\mathrm{CH}$ II. In addition, if we choose $\alpha=0.25$, the probabilities of perfect timing synchronization are approximately equal to 0.5 in all channel models. The reason why the scheme in [17] has poor performance in timing synchronization are that AWGN affects the entire fine time adjustment process at low SNR and wide interval for fine time adjustment, especially in $\mathrm{CH}$ I and $\mathrm{CH}$ II. In addition, pre-simulations for searching a proper $\alpha$ are also needed. Therefore, low SNR and wide interval for fine time adjustment significantly degrade the performance in $[9,10,12,13]$. In Figures 5 and 6 , our proposed approach has better performance than the joint scheme [17]. In Figure 5, our proposed approach performs almost unbiased at any low SNR, and wide interval for fine time adjustment in [17] leads the time estimator to have nonzero biases. In Figure 6, the proposed approach achieves zero RMSE when SNR exceeds $1 \mathrm{~dB}$ due to the ability to identify the first arrival path in the channel; however, the joint scheme still has large RMSE of time estimators [17].
Moreover, the performance of channel estimation is shown in Figures 7 and 8. The performance of channel estimation is evaluated after the estimated timing offset is obtained. In Figures 7 and 8, the proposed approach has a consistent and better performance of channel estimation for $2 \times 2$ and $3 \times 3$ MIMO systems, and the joint scheme has a poor performance of channel estimation because of incorrect estimated timing offsets [17]. In addition, the performance of the proposed approach is close to the Cramér-Rao bound [21].

\section{Conclusions}

In this paper, we have developed a joint timing synchronization and channel estimation scheme for centralized multiple-input communications systems based on a well-designed training sequence arrangement. The coarse timing and frequency offset estimation in our proposed approach have excellent performance at low SNR. In addition, a generalized maximum-likelihood channel estimation scheme is presented. Based on the generalized ML estimation scheme, different CIRs from all transmit antennas can be obtained. We also developed a fine time adjustment algorithm to find the actual position of the first arrival path in LOS and NLOS channel models. Based on simple thresholds without any pre-simulation 


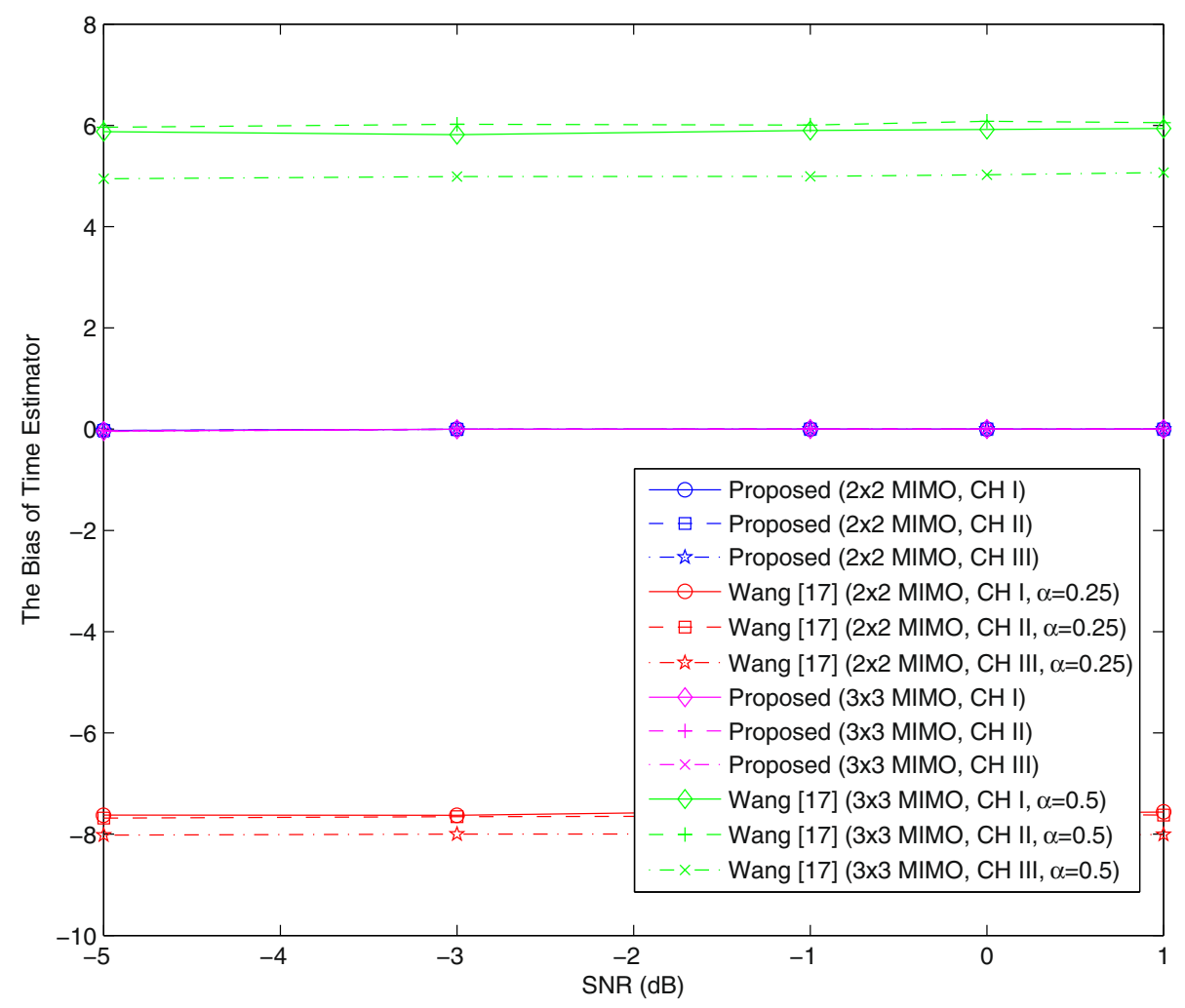

Figure 5 The bias of timing estimator, $E[\hat{\tau}-\tau]$, where $E[\cdot]$ is the expectation function.

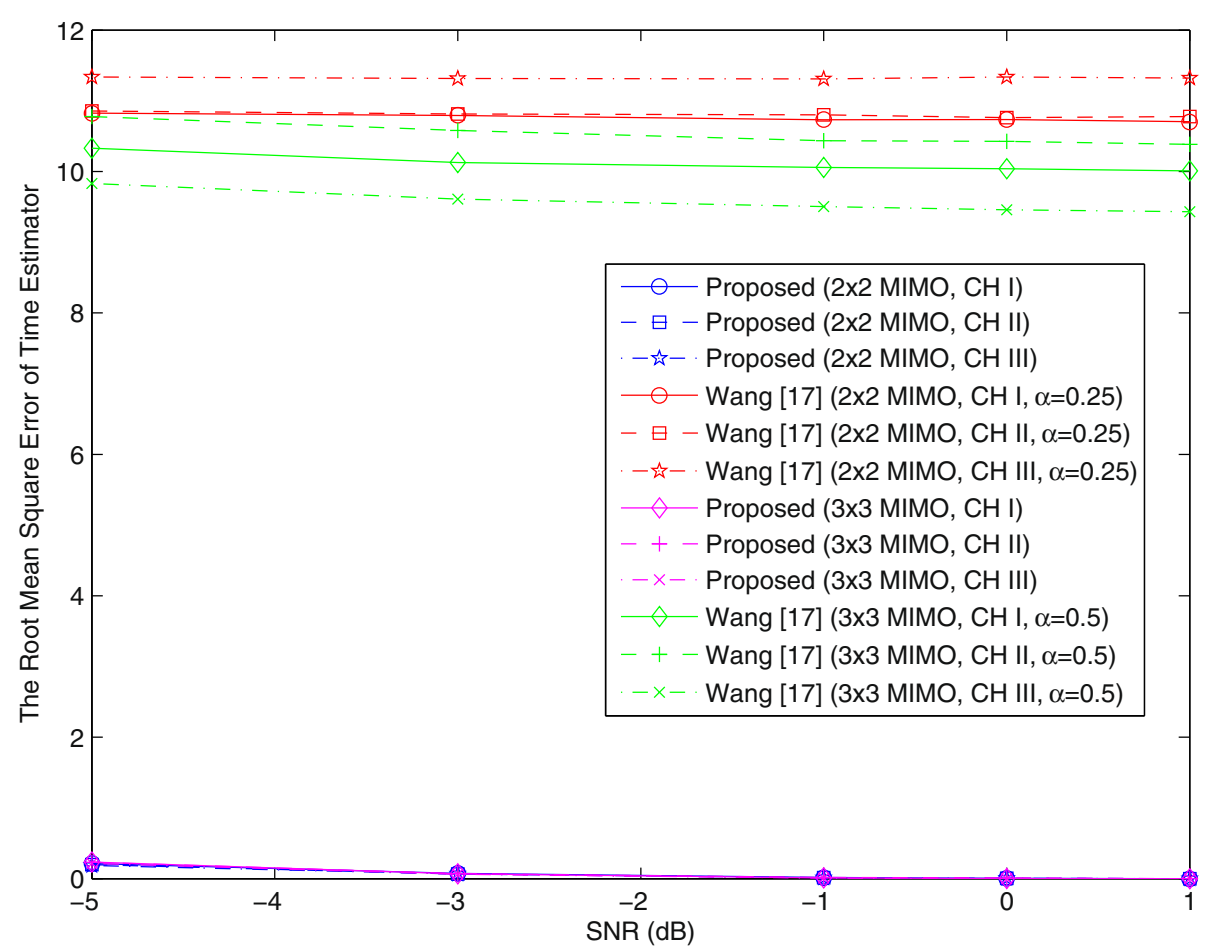

Figure 6 The root mean squared error of timing estimator, $\sqrt{E\left[|\hat{\tau}-\tau|^{2}\right]}$. 


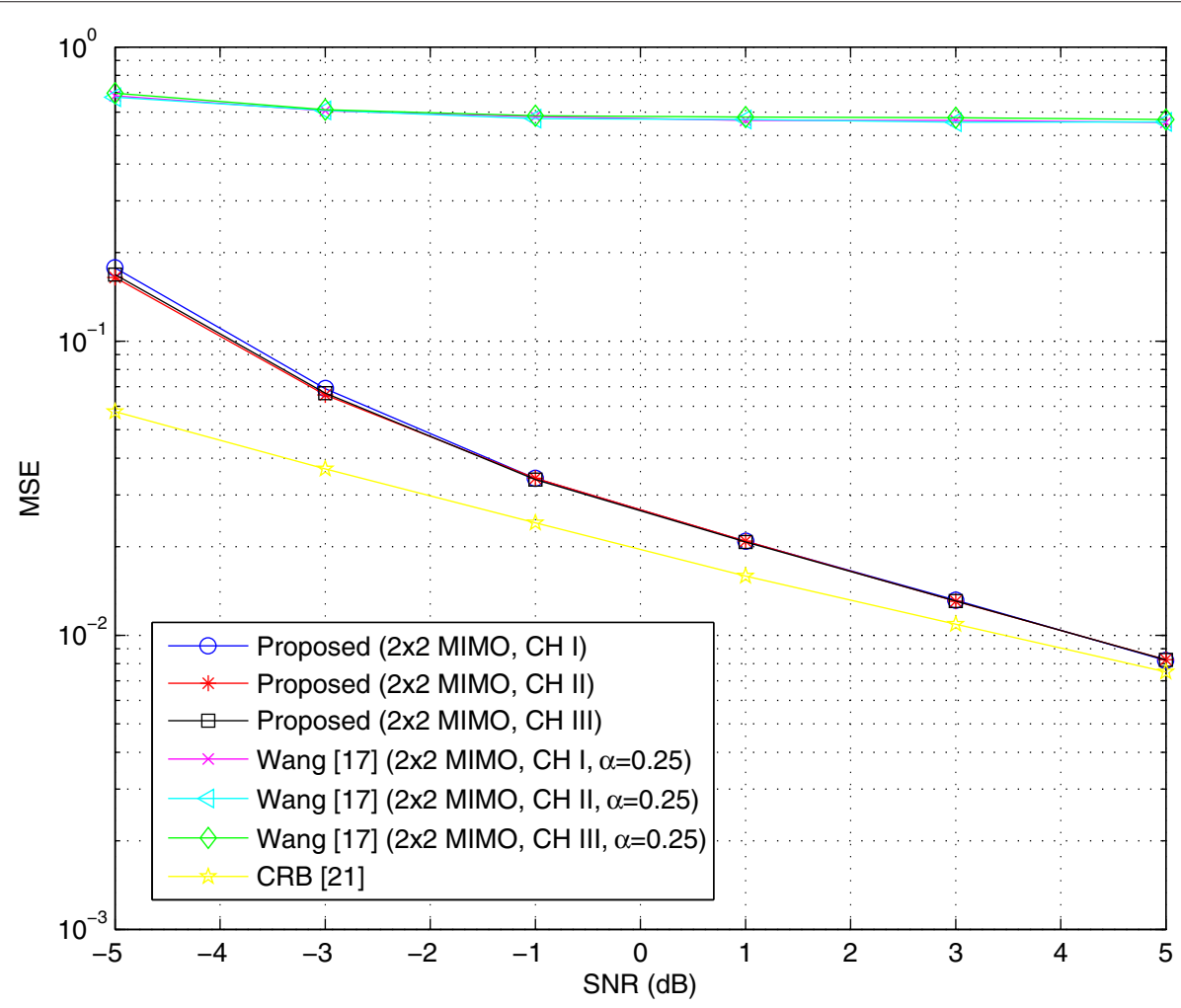

Figure 7 The MSE of CIR estimates for a $2 \times 2$ MIMO system, $E\left[\left|\hat{h}_{b}-\mathbf{h}_{b}\right|^{2}\right]$.

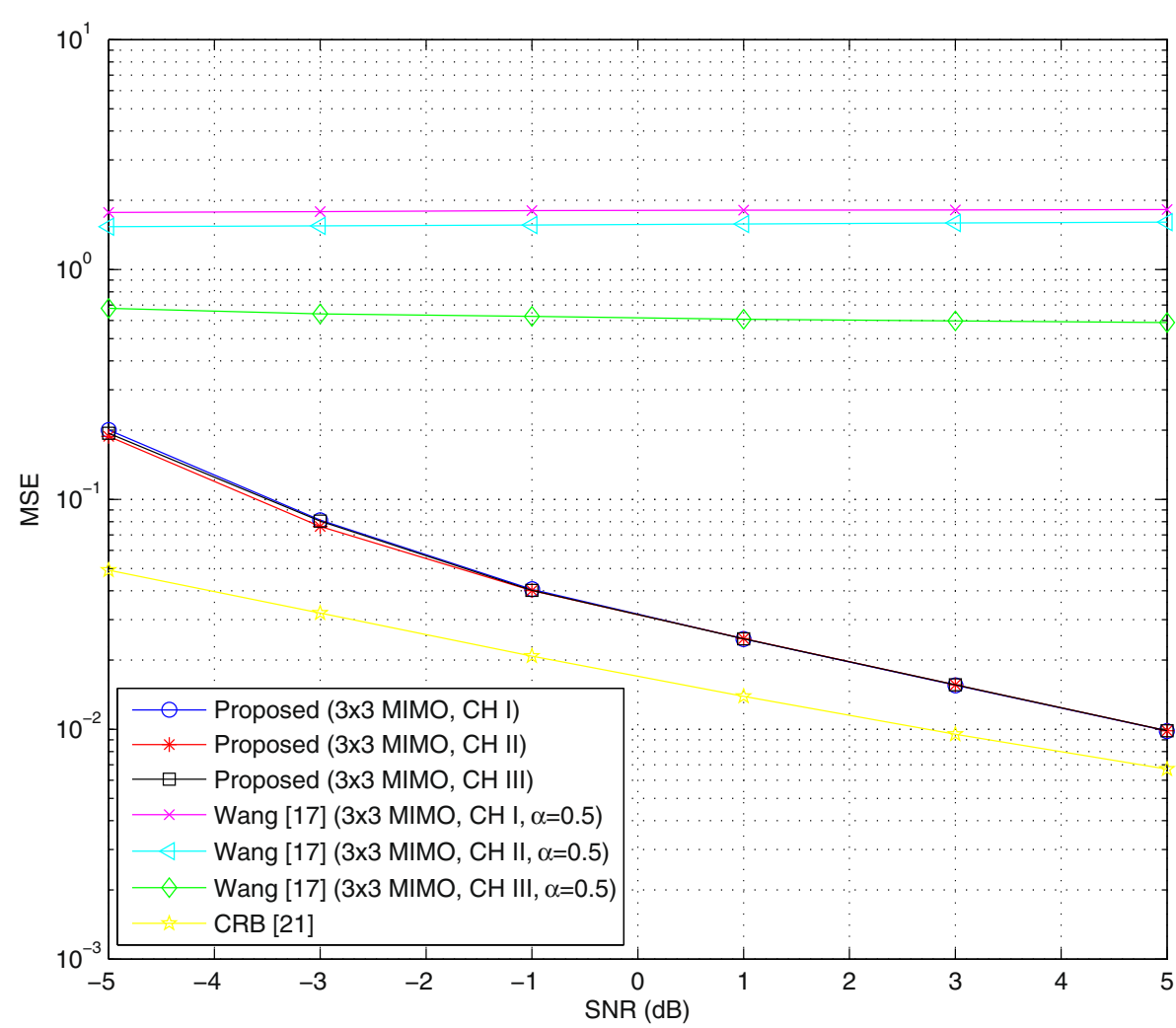

Figure 8 The MSE of CIR estimates for a 3 × 3 MIMO system. 
and theoretical derivation, the proposed fine time adjustment algorithm outperforms conventional schemes even in a very low SNR environment. Simulation results show that there are no timing errors in our proposed time estimator when SNR exceeds 0dB. Finally, the proposed approach achieves excellent performance in channel estimation, because the proposed time estimator outperforms the existing methods.

\section{Competing interests}

Both authors declare that they have no competing interests.

Received: 14 April 2013 Accepted: 11 August 2013

Published: 22 August 2013

\section{References}

1. G Raleigh, J Cioffi, Spatio-temporal coding for wireless communications. IEEE Trans. Commun. 46(3), 357-366 (1998)

2. A Moustakas, S Simon, Optimized multiple-input single-output (MISO) communication systems with general Gaussian channels: nontrivial covariance and nonzero mean. IEEE Trans. Inf. Theory. 49(10), 2770-2780 (2003)

3. R van Nee, R Prasad, OFDM Wireless Multimedia Communication (Artech House, Norwood, 2004)

4. G Stüber, J Barry, S Mclaughlin, Y Li, M Ingram, T Pratt, Broadband MIMO-OFDM wireless communications. Proc. IEEE. 92(2), 271-294 (2004)

5. T Pollet, M van Bladel, M Moeneclaey, BER sensitivity of OFDM systems to carrier frequency offset and Wiener phase noise. IEEE Trans. Commun. 43, 191-193 (1995)

6. J van de Beek, M Sandell, P Börjesson, ML estimation of time and frequency offset in OFDM systems. IEEE Trans. Signal Process. 45(7), 1800-1805 (1997)

7. T Schmidl, D Cox, Robust frequency timing synchronization for OFDM. IEEE Trans. Commun. 45(12), 1613-1621 (1997)

8. H Minn, M Zeng, $\vee$ Bhargava, On timing offset estimation for OFDM systems. IEEE Commun. Lett. 4(7), 242-244 (2000)

9. H Minn, V Bhargava, K Letaief, A robust timing and frequency synchronization for OFDM systems. IEEE Trans. Wireless Commun. 2(4), 822-839 (2003)

10. H Minn, V Bhargava, K Letaief, A combined timing and frequency synchronization and channel estimation for OFDM. IEEE Trans. Commun 54(3), 416-422 (2006)

11. C-L Wang, H-C Wang. A low-complexity joint timing synchronization and channel estimation scheme for orthogonal frequency division multiplexing systems, in IEEE International Conference on Communications (Istanbul, 11-15 June 2006), pp. 5670-5675, (IEEE, Piscataway, 2006)

12. C-L Wang, H-C Wang. An optimized joint timing synchronization and channel estimation scheme for OFDM systems, in Proceedings of the IEEE Vehicular Technology Conference, (Singapore, 11-14 May 2008), pp. 908-912, (IEEE, Piscataway, 2008)

13. C-L Wang, H-C Wang, On joint fine time adjustment and channel estimation for OFDM systems. IEEE Trans. Wireless Commun. 8(10), 4940-4944 (2009)

14. J-H Wen, G-R Lee, J-W Liu, T-L Kung. Joint frame synchronization and channel estimation scheme using regression method in OFDM systems, in Proceedings of the IEEE International Wireless Communications, Networking and Mobile Computing, (Shanghai, 21-25 Sept 2007), pp. 164-167, (IEEE, Piscataway, 2007)

15. A Mody, G Stüber. Synchronization for MIMO OFDM systems, in Proceedings of the IEEE Global Telecommunications Conference, (San Antonio, 25-29 Nov 2001), pp. 509-513, (IEEE, Piscataway, 2001)

16. N Long, H Park. Joint fine time synchronization and channel estimation for MIMO-OFDM WLAN, in Proceedings of the IEEE International Symposium on Intelligent Signal Processing and Communication Systems, (Seoul, 18-19 Nov 2004), pp. 463-467, (IEEE, Piscataway, 2004)

17. H-C Wang, C-L Wang. A new joint time synchronization and channel estimation scheme for MIMO-OFDM systems, in Proceedings of the IEEE Global Telecommunications Conference, (San Francisco, 27 Nov 1 Dec 2006), pp. 1-5, (IEEE, Piscataway, 2006)
18. C-L Wang, H-C Wang, Optimized joint fine timing synchronization and channel estimation for MIMO systems. IEEE Trans. Commun. 59(4), 1089-1098 (2011)

19. T-L Kung, KK Parhi, Optimized joint timing synchronization and channel estimation for OFDM systems. IEEE Wireless Commun. Lett. 1(3), 149-152 (2012)

20. IEEE std 802.11. Wireless LAN medium access control (MAC) and physical layer (PHY) specifications (IEEE, Piscataway, 2012)

21. P Stoica, O Besson, Training sequence design for frequency offset and frequency-selective channel estimation. IEEE Trans. Commun. 51(11), 1910-1917 (2003)

doi:10.1186/1687-6180-2013-139

Cite this article as: Kung and Parhi: Optimized joint timing synchronization and channel estimation for communications systems with multiple transmit antennas. EURASIP Journal on Advances in Signal Processing 2013 2013:139.

\section{Submit your manuscript to a SpringerOpen ${ }^{\mathcal{O}}$ journal and benefit from:}

- Convenient online submission

- Rigorous peer review

- Immediate publication on acceptance

- Open access: articles freely available online

- High visibility within the field

- Retaining the copyright to your article

Submit your next manuscript at $>$ springeropen.com 